\title{
Why Are the Effective Equations of Loop Quantum Cosmology so Accurate?
}

\author{
Carlo Rovelli ${ }^{1,2, *}$ and Edward Wilson-Ewing, \\ ${ }^{1}$ Aix Marseille Université, CNRS, CPT, UMR 7332, 13288 Marseille, France \\ ${ }^{2}$ Université de Toulon, CNRS, CPT, UMR 7332, 83957 La Garde, France \\ ${ }^{3}$ Department of Physics and Astronomy, Louisiana State University, Baton Rouge, 70803, USA
}

\begin{abstract}
We point out that the relative Heisenberg uncertainty relations vanish for non-compact spaces in homogeneous loop quantum cosmology. As a consequence, for sharply peaked states quantum fluctuations in the scale factor never become important, even near the bounce point. This shows why quantum back-reaction effects remain negligible and explains the surprising accuracy of the effective equations in describing the dynamics of sharply peaked wave packets. This also underlines the fact that minisuperspace models — where it is global variables that are quantized - do not capture the local quantum fluctuations of the geometry.
\end{abstract}

PACS numbers: 98.80.Qc

\section{INTRODUCTION}

The loop quantum cosmology (LQC) effective equations provide quantum-gravity corrections to the classical solutions of the Friedmann cosmologies [1, 2], but they are expected to break down when quantum gravity effects become stronger. This expectation arises since quantum fluctuations of the geometry are expected to become important as the space-time curvature nears the Planck scale, and when quantum fluctuations become large, quantum back-reaction could become important [3]. However, despite this expectation, numerical evidence shows that the effective equations (even when neglecting all quantum back-reaction effects) provide an excellent approximation to the full dynamics of sharply peaked states, including at the bounce point where quantum gravity effects are strongest [4, 5]. In this sense, the effective equations are surprisingly accurate in the Planck regime. Why is quantum back-reaction negligible in the effective dynamics?

A closely related question concerns the magnitude of quantum fluctuations of the geometry at the bounce. Naively, one could expect classical space-time to become ill-defined at the bounce, because of the dominance of quantum gravity effects that may cause large quantum fluctuations. But this is not necessarily the case: LQC predicts bouncing geometries where quantum fluctuations of the scale factor at the bounce can be arbitrarily small. How is this possible?

In this paper we offer an explanation of these facts, which we believe may shed some light on the foundations of LQC (and minisuperspace quantum cosmology in general). For the sake of simplicity we focus on the case of a flat Friedmann-Lemaître-Robertson-Walker (FLRW) universe with a perfect fluid of constant equation of state. It is easy to generalize the discussion to other matter fields and also to other homogeneous cosmologies. We

*Electronic address: rovelli@cpt.univ-mrs.fr

${ }^{\dagger}$ Electronic address: wilson-ewing@phys.lsu.edu consider states which, far away from the Planck regime, admit a clear semi-classical interpretation, i.e., states that are sharply peaked in both the configuration and momentum variables.

\section{HAMILTONIAN FORMULATION OF COSMOLOGY}

In loop quantum cosmology, the fundamental variables are taken to be the Ashtekar-Barbero connection $A_{a}^{i}$ and the densitized triad $E_{i}^{a}$, which in the flat FLRW spacetime can be parametrized as [6]

$$
A_{a}^{i}=\tilde{c}\left(\mathrm{~d} x^{i}\right)_{a}, \quad E_{i}^{a}=\tilde{p} \sqrt{\dot{q}}\left(\frac{\partial}{\partial x^{i}}\right)^{a},
$$

where $\tilde{p}=a(t)^{2}$, with $a(t)$ being the usual scale factor in the FLRW model (and ignoring a possible sign factor due to the orientation of the triads), and $x^{i}$ are Cartesian coordinates on the spatial manifold defining a fiducial spatial metric

$$
\mathrm{d} \stackrel{\circ}{ }^{2}=\left(\mathrm{d} x^{1}\right)^{2}+\left(\mathrm{d} x^{2}\right)^{2}+\left(\mathrm{d} x^{3}\right)^{2},
$$

with determinant $\stackrel{\circ}{q}=1$.

It is possible to rescale the fiducial coordinates by a factor $\alpha, x^{i} \rightarrow \alpha x^{i}$ in which case $\stackrel{\circ}{q} \rightarrow \alpha^{6} \stackrel{\circ}{q}$ and

$$
\tilde{c} \rightarrow \alpha^{-1} \tilde{c}, \quad \tilde{p} \rightarrow \alpha^{-2} \tilde{p},
$$

so that $A_{a}^{i}$ and $E_{i}^{a}$ remain invariant.

Fix a space-time region $\mathcal{V}$, called the fiducial cell, with fiducial-metric volume $V_{o}=\int_{\mathcal{V}} \sqrt{q}$. Inserting the form (11) for the variables $A_{a}^{i}$ and $E_{i}^{a}$, the Holst action [7] for this region is given by the constraints plus the "symplectic" term

$$
\frac{1}{8 \pi G \gamma} \int_{\mathcal{V}} \dot{A}_{a}^{i} E_{i}^{a} \mathrm{~d}^{3} x=\frac{3 V_{o}}{8 \pi G \gamma} \dot{\tilde{c}} \tilde{p}
$$

where $\gamma$ is the Immirzi parameter. Notice that if space is non-compact, for the theory to be well-defined, it is 
necessary to introduce the fiducial cell $\mathcal{V}$ in order to avoid the divergence of this integral (and also the integrals that appear in the constraint functions). In this sense, $\mathcal{V}$ acts as an infrared regulator.

It follows that the fundamental Poisson bracket in the gravitational sector is

$$
\{\tilde{c}, \tilde{p}\}=\frac{8 \pi G \gamma}{3 V_{o}}
$$

This Poisson bracket is invariant under the rescaling (3), but it does depend on the choice of the fiducial cell $\mathcal{V}$, as emphasized in [8]. This dependence does not affect the classical dynamics, but it does play a very important (though subtle) role in the quantum theory.

The dynamics is given by the Hamiltonian constraint $\mathcal{C}_{H}$. Taking the lapse $N=1$, and in the presence of a massless scalar field $\tilde{\phi}$ whose Poisson bracket with its conjugate momentum is $\left\{\tilde{\phi}, \tilde{\pi}_{\phi}\right\}=V_{o}^{-1}, \mathcal{C}_{H}$ is [4]

$$
\mathcal{C}_{H}=\int_{\mathcal{V}} N \mathcal{H}=-\frac{3 V_{o} \sqrt{\tilde{p}} \tilde{c}^{2}}{8 \pi G \gamma^{2}}+V_{o} \frac{\tilde{\pi}_{\phi}^{2}}{2 \tilde{p}^{3 / 2}} \approx 0 .
$$

From $\dot{\mathcal{O}}=\left\{\mathcal{O}, \mathcal{C}_{H}\right\}$, it follows that the classical dynamics is independent from the fiducial cell: $V_{o}$ appears linearly in the Hamiltonian constraint and is cancelled by the $1 / V_{o}$ factor in the Poisson bracket (5).

Since the classical dynamics is independent of the choice of the fiducial cell, there is no need to remove the "infrared regulator" provided by the choice of the cell. In other words, the removal of the regulator in the classical theory is trivial since the dynamics is not affected by the choice of $\mathcal{V}$.

\section{QUANTUM COSMOLOGY}

In the quantum theory, the Poisson brackets are replaced by commutators and the variables become operators. In order to gain some intuition, we shall start by considering Wheeler-DeWitt (WDW) quantum cosmology as an example. In WDW theory, the basic commutator is given by

$$
[\widehat{\tilde{c}}, \widehat{\tilde{p}}]=i \frac{8 \pi G \hbar \gamma}{3 V_{o}},
$$

and at first sight, it seems as though the quantum theory could also be independent from the choice of $\mathcal{V}$ since in the Heisenberg picture

$$
\frac{\mathrm{d} \widehat{\mathcal{O}}}{\mathrm{d} t}=\left[\widehat{\mathcal{O}}, \widehat{\mathcal{C}_{H}}\right]
$$

and again the numerical factors of $V_{o}$ cancel out.

But the quantum theory does more than just giving the dynamics: it also determines the quantum fluctuations of the classical variables. The commutation relation (7) implies the uncertainty relation

$$
\Delta \tilde{c} \cdot \Delta \tilde{p} \geq \frac{4 \pi G \hbar \gamma}{3 V_{o}} .
$$

This time nothing cancels the $V_{o}$ factor. Therefore the choice of the quantisation region, i.e., of the fiducial cell, affects the quantum theory. (Another way to see that the quantum theory is not invariant under a rescaling of the fiducial cell is by explicitly determining how the expectation values of a given state change for different choices of $V_{o}$ [8].)

In fact, by taking $V_{o}$ arbitrarily large, the right hand side of the Heisenberg uncertainty relations (9) vanishes. Clearly, the quantum fluctuations can then become arbitrarily small. This does not mean that all states must have small quantum fluctuations, but it means that it is possible to construct states with arbitrarily small uncertainties in $\tilde{c}$ as well $\tilde{p}$.

The minimum possible amplitude of quantum fluctuations is a physical quantity. If we require it to be independent of the fiducial cell, because we want to view $\mathcal{V}$ just as an infrared regulator, we can take the limit of $V_{o} \rightarrow \infty$. This is routinely done in quantum cosmology. This gives

$$
\lim _{V_{o} \rightarrow \infty} \Delta \tilde{c} \Delta \tilde{p} \geq 0
$$

showing that there is no minimal non-zero amplitude for quantum fluctuations in quantum cosmology, at least for non-compact homogeneous spaces. Thus, it is possible to build states whose quantum fluctuations are always arbitrarily small.

It is clear that since the classical symplectic structure in LQC is the same as for WDW quantum cosmology, similar arguments will apply for LQC as well, and this explains why the effective equations of LQC are so accurate. Even more, it is possible to go further and show that for large $V_{o}$, states (in the Schrödinger picture) whose quantum fluctuations are small at one time continue to have small quantum fluctuations at all times, including at the bounce point. This result implies (for large $V_{o}$ ) that since the quantum fluctuations remain small, the effective Hamiltonian is expected to give an excellent approximation to the full quantum dynamics at all times, including the bounce point.

In order to show this in LQC, it is convenient to change variables to [9]

$$
\tilde{\beta}=\frac{\tilde{c}}{\sqrt{\tilde{p}}}, \quad \tilde{V}=\tilde{p}^{3 / 2},
$$

whose Poisson bracket, in the classical theory as well as the effective theory, is

$$
\{\tilde{\beta}, \tilde{V}\}=\frac{4 \pi G \gamma}{V_{o}} .
$$

In LQC, all expressions that contain the connection must be expressed in terms of holonomies. Because of this, there is no operator corresponding to $\tilde{\beta}$; rather the operators of interest are complex exponentials (or trigonometric functions) of $\ell \tilde{\beta}$, which correspond to holonomies of the Ashtekar-Barbero connection along 
edges of physical length equal to $\ell$. In particular, in the Hamiltonian constraint operator the field strength operator is expressed in terms of holonomies around a minimal area loop with $\ell=\lambda$, where $\lambda^{2} \sim \ell_{\mathrm{Pl}}^{2}$ is the area gap, the smallest non-zero eigenvalue of the area operator in loop quantum gravity [4]. As shall be seen below, this input appears in the resulting LQC effective theory.

Now we shall briefly recall the main ingredients of LQC that will be necessary here, for more details see [4, 9]. A convenient basis for the LQC of the flat FLRW spacetime are eigenstates of the volume operator,

$$
\widehat{\tilde{V}}|\tilde{V}\rangle=\tilde{V}|\tilde{V}\rangle, \quad \text { with } \quad\left\langle\tilde{V}_{1} \mid \tilde{V}_{2}\right\rangle=\delta_{\tilde{V}_{1}, \tilde{V}_{2}} .
$$

The other basic operators in the gravitational sector of LQC are the holonomy (also called shift) and inverse volume operators, defined as

$$
\begin{aligned}
\widehat{e^{-i \ell \tilde{\beta}}}|\tilde{V}\rangle & =\left|\tilde{V}+4 \pi G \hbar \gamma \ell / V_{o}\right\rangle, \\
\widehat{\tilde{V}^{-1}}|\tilde{V}\rangle & = \begin{cases}0 & \text { if } \tilde{V}=0, \\
\tilde{V}^{-1}|\tilde{V}\rangle & \text { otherwise. }\end{cases}
\end{aligned}
$$

Note that there exists a large number of ambiguities in the definition of inverse volume operators (see e.g. Sec. IV in [10] for a more detailed discussion on this point), here we choose (15) both for its simplicity, and because it is the only known inverse volume operator in LQC that does not depend on the choice of the fiducial cell.

From (13) and (14), it follows that the basic commutator in $\mathrm{LQC}$ is

$$
\left[\widehat{\tilde{V}}, \widehat{e^{-i \ell \tilde{\beta}}}\right]=\frac{4 \pi G \hbar \gamma \ell}{V_{o}} \widehat{e^{-i \ell \tilde{\beta}}} .
$$

At this point, it is possible to define the Hamiltonian constraint operator in LQC and then study the resulting quantum dynamics, as in [4, 9].

Instead, here we shall use the effective equations and, assuming a state that is initially sharply peaked in both its configuration and momentum variables, determine when quantum fluctuations become comparable to expectation values. It is at this point that quantum backreaction will become important and the effective theory can no longer be trusted. Thus, the effective theory itself will tell where it breaks down.

In the $(\tilde{V}, \tilde{\beta})$ variables, the LQC effective theory is determined by the effective Hamiltonian constraint [1]

$$
\mathcal{C}_{H}=-\frac{3 V_{o} \tilde{V}}{8 \pi G \gamma^{2} \lambda^{2}} \sin ^{2}(\lambda \tilde{\beta})+V_{o} \tilde{V} \rho \approx 0,
$$

for a generic perfect fluid. The continuity equation is unchanged in the effective theory of LQC (recall $\tilde{V}=a^{3}$ )

$$
\frac{\mathrm{d} \rho}{\mathrm{d} t}+\frac{1}{\tilde{V}} \frac{\mathrm{d} \tilde{V}}{\mathrm{~d} t}(\rho+P)=0 .
$$

Assuming a constant equation of state $P=\omega \rho$ with $-1 \leq$ $\omega \leq 1$,

$$
\rho=\frac{\tilde{\rho}_{o}}{\tilde{V}^{n}}, \quad 0 \leq n \leq 2,
$$

with $n=1+\omega$. Note that since $\rho$ is independent of $V_{o}$, $\tilde{\rho}_{o}$ is as well.

The commutator (16) implies the uncertainty relation

$$
\Delta \tilde{V} \cdot \Delta\left(\frac{\sin \lambda \tilde{\beta}}{\lambda}\right) \geq \frac{2 \pi G \hbar \gamma}{V_{o}} \cdot|\langle\cos \lambda \tilde{\beta}\rangle|,
$$

and from this relation it is possible to calculate when quantum fluctuations become important. The constraint (17) determines the value of $\tilde{V}$ when the bounce occurs in the effective theory and so it is possible to check whether the quantum fluctuations become important before the bounce occurs or not.

Hamilton's equation for $\tilde{V}$ from (17) is

$$
\frac{\mathrm{d} \tilde{V}}{\mathrm{~d} t}=\frac{3 \tilde{V}}{\gamma \lambda} \sin (\lambda \tilde{\beta}) \cos (\lambda \tilde{\beta}) ;
$$

this equation of motion can be solved by squaring it and then using the constraint equation $\mathcal{C}_{H}=0$, giving

$$
\tilde{V}(t)=\left(6 \pi G \tilde{\rho}_{o} n^{2}\left(t-t_{o}\right)^{2}+\frac{\tilde{\rho}_{o}}{\rho_{c}}\right)^{1 / n},
$$

where the critical energy density is $\rho_{c}=3 /\left(8 \pi G \gamma^{2} \lambda^{2}\right)$. The resulting $\tilde{V}_{\text {bounce }}$ is independent of $V_{o}$,

$$
\tilde{V}_{\text {bounce }}=\left(\frac{\tilde{\rho}_{o}}{\rho_{c}}\right)^{1 / n}
$$

Now let us determine the volume where initially small quantum fluctuations become important. $\mathcal{C}_{H}=0$ implies

$$
\frac{\sin \lambda \tilde{\beta}}{\lambda}=\frac{\sqrt{\tilde{\rho}_{o}}}{\lambda \sqrt{\rho_{c}}} \cdot \frac{1}{\tilde{V}^{n / 2}} .
$$

Then, as $\Delta[f(x)]=\Delta(x) \cdot\left|\partial_{x} f(x)\right|$ for small $\Delta x$, we find that $^{1}$

$$
\Delta\left(\frac{\sin \lambda \tilde{\beta}}{\lambda}\right)=\frac{n \sqrt{\tilde{\rho}_{o}}}{2 \lambda \sqrt{\rho_{c}}} \frac{\Delta \tilde{V}}{\tilde{V}^{n / 2+1}} .
$$

Note that here we are only considering states that are initially sharply-peaked, by which we mean that initially the relative uncertainties $\Delta \tilde{V} / \tilde{V}$ and $\Delta(\sin \lambda \tilde{\beta}) / \sin \lambda \tilde{\beta}$ are small compared to 1 , and that the higher-order moments are smaller still.

Assuming that the uncertainty relation (20) is nearly saturated and using (25), we find

$$
\frac{(\Delta \tilde{V})^{2}}{\tilde{V}^{n / 2+1}} \sim \frac{4 \pi G \hbar \gamma \lambda}{n V_{o}} \sqrt{\frac{\rho_{c}}{\tilde{\rho}_{o}}}|\cos \lambda \tilde{\beta}| .
$$

\footnotetext{
1 Here we are neglecting the fluctuations in the matter field. This is because the matter field is typically used as a relational clock in LQC and so acts as a parameter with respect to which other observables are measured. Note that the qualititative result of this calculation is not affected if we include matter fluctuations as well.
} 
Quantum fluctuations become important when $\tilde{V} \sim$ $\Delta \tilde{V}$, which occurs for

$$
\tilde{V}_{\mathrm{qf}} \sim\left(\frac{4 \pi G \hbar \gamma \lambda}{n V_{o}} \sqrt{\frac{\rho_{c}}{\tilde{\rho}_{o}}}|\cos \lambda \tilde{\beta}|\right)^{\frac{2}{2-n}},
$$

and we immediately see that, for large $V_{o}$,

$$
\tilde{V}_{\text {bounce }} \gg \tilde{V}_{\mathrm{qf}} \text {. }
$$

An analogous calculation can be performed to determine $\sin (\lambda \tilde{\beta})_{\text {bounce }}$ and $\sin (\lambda \tilde{\beta})_{\mathrm{qf}}$, this gives

$$
\sin (\lambda \tilde{\beta})_{\text {bounce }}=1,
$$

and

$$
\sin (\lambda \tilde{\beta})_{\mathrm{qf}} \sim V_{o}^{\frac{n}{2-n}}
$$

where we have only written the $V_{o}$ dependence for $\sin (\lambda \tilde{\beta})_{\mathrm{qf}}$. Clearly, as $2-n \geq 0$, it is impossible for $\sin (\lambda \tilde{\beta})_{\mathrm{qf}}$ to be reached if $V_{o}$ is taken to be sufficiently large.

Thus, it is clear that, for large $V_{o}$, states that are initially sharply peaked will remain sharply peaked throughout their evolution as they bounce before quantum fluctuations have a chance to become important, and the effective Hamiltonian will provide an excellent approximation to the full quantum dynamics at all times.

Of course, it is important to keep in mind that for states that are not sharply peaked, the effective equations are not a good approximation as can be seen explicitly in [11]. In addition, for small $V_{o}$, we see that $\tilde{V}_{\mathrm{qf}}$ may be larger than $\tilde{V}_{\text {bounce }}$ in which case the effective dynamics generated by the effective Hamiltonian (17) cannot be trusted for $\tilde{V} \lesssim \tilde{V}_{\text {qf }}$. Thus, the effective equations may fail for states that are not sharply peaked, or where $V_{o}$ is small.

Very similar calculations yield the same results for compact models: the effective equations provide an excellent approximation to the full quantum dynamics for sharply peaked states so long as $V_{o}$ is sufficiently large. [One difference in the calculation is that in a compact space, it is not necessary to introduce a fiducial cell as the integrals (4) and (6) are bounded and so do not diverge. Then, $V_{o}$ corresponds to the volume of the entire space with respect to $\stackrel{\circ}{q}_{a b}$ and therefore $V_{o}$ is no longer a free parameter but rather is fixed. Another point is that other inverse triad operators than (15) may be chosen in compact spaces; while this would complicate the calculations, the qualitative results should remain unchanged. Other than these points, the calculation for the compact space is essentially identical to the one given here for a non-compact space.] Thus, we expect sharply peaked states in compact spaces to also remain sharply peaked at all times, including at the bounce point, so long as $V_{o}$ is sufficiently large. On the other hand, for small $V_{o}$, quantum fluctuations are expected to become important before the bounce occurs, in which case the effective equations may no longer provide a good approximation to the full quantum dynamics.

Now it is clear why the effective equations are so accurate, even in the deep Planck regime: in order to build a semi-classical state, fluctuations must be small in the classical limit. Since there is no lower bound on the amplitude of quantum fluctuations in the theory, it is possible to choose states where the quantum fluctuations are arbitrarily small, and so never become important, even at the bounce point.

It has been pointed out that, in general, quantum backreaction from higher order moments must be included in the effective equations [3], but quantum back-reaction can safely be ignored so long as quantum fluctuations are negligible. As we have seen here, there exists a large family of solutions (i.e., solutions with initially small fluctuations and sufficiently large $V_{o}$ ) where ignoring quantum back-reaction is a valid approximation.

This is also why the fluctuations in the scale factor and other large-scale observables can be negligible: for non-compact spaces in LQC (and also WDW and other quantum cosmology minisuperspace models) it is always possible, in the limit $V_{o} \rightarrow \infty$, for the fluctuations to be arbitrarily small.

These considerations answer the two questions we asked in the introduction. Still, the answer is a little disconcerting. What is the sense of a quantum theory where fluctuations can always be reduced to zero? Isn't this in contradiction with standard quantum theory ideas, and the irreducibility of the Heisenberg relations?

\section{WHERE ARE THE FLUCTUATIONS?}

A source of confusion in searching for a description of the quantum fluctuations in cosmology is due to a common misinterpretation of the theory. When we restrict the space of the solutions of the classical theory to the homogenous fields (11), and we consider only their dynamics, we are effectively disregarding the quantum theory of all of the higher modes of the field. The commutation relations (7) show that the larger modes behave effectively as averages of local variables.

An analogy of this situation is provided by the following example. Consider a material formed by $N$ atoms, each having unit mass. Let the position $x_{n}$ and momentum $p_{n}=\dot{x}_{n}$ of each atom satisfy standard commutation relations $\left[x_{n}, \dot{x}_{n}\right]=i \hbar$. The center of mass of the system

$$
X=\frac{1}{N} \sum_{n} x_{n}
$$

and its velocity $\dot{X}$ satisfy the commutation relation

$$
[X, \dot{X}]=\frac{i \hbar}{N},
$$

which goes to zero as $N$ goes to infinity. Thus the centre of mass effectively behaves classically when there are 
many particles. On physical grounds, this obviously does not imply that the quantum fluctuations of the individual atoms go to zero in the large $N$ limit. It only implies that the centre of mass is blind to these. A more general discussion on the classical limit of macroscopic (large $N$ ) observables can be found in [12].

A moment of reflection shows that the mathematics of quantum cosmology is analogous. The homogenous variables are the lowest modes in a Fourier expansion and are therefore like averages of the local variables, namely the fields at a point. As before, the fact that the commutation relations (7) vanish in the large $V_{o}$ limit does not imply at all that the quantum fluctuation of the fields at a point go to zero. It only shows that $\tilde{\beta}$ and $\tilde{V}$ are blind to these. In other words, homogeneous quantum cosmology is blind to the local quantum fluctuations of the gravitational field.

The theory with a given fiducial cell $\mathcal{V}$, in other words, describes only the quantum effects on the modes of the size of $\mathcal{V}$, and not the smaller modes. Different choices of $\mathcal{V}$ do not lead to a mathematical lack of definiteness: they correspond to analyzing different modes of the theory. For example, in order to study the dynamics of the largescale structure of the universe, it is appropriate to choose a very large fiducial cell, in which case quantum fluctuations will be negligible for sharply-peaked states. On the other hand, we do expect quantum fluctuations to necessarily be important for the physics at trans-Planckian scales. We will discuss this last point further in Sec. D

To know how the geometry fluctuates at short scales, we can simply take $\mathcal{V}$ to be small rather than large. It is this that provides a description of local quantum fluctuations. Let us sketch here what this implies.

To do this calculation, it is convenient to use the variables

$$
V=V_{o} \tilde{V}, \quad \beta=\tilde{\beta},
$$

where $V$ corresponds to the physical volume of the fiducial cell [4, 9].

As an aside, note that this rescaling is akin to using the centre of mass momentum $P=N \dot{X}$ in 32 rather than the velocity. In this case the basic commutator is $[X, P]=i \hbar$, which is independent of $N$. Of course, using different variables does not change the underlying physics in the many-body example (or in LQC) and the centre of mass variable effectively behaves classically for large $N$, whether one uses the variable $\dot{X}$ or $P$.

Similarly, the Poisson bracket in the new variables is given by $\{V, \beta\}=4 \pi G \gamma$ and therefore the uncertainty relations in the quantum theory are independent of $V_{o}$. Nonetheless, we can easily show that the same result holds. The basic uncertainty relation in terms of the new variables is

$$
\Delta V \cdot \Delta(\sin \lambda \beta) \geq 2 \pi G \hbar \gamma \lambda \cos \lambda \beta
$$

In order to bound the relative uncertainties, we divide both sides by $V \sin \lambda \beta$, and on the right-hand side use the relation $\sin \lambda \beta=\sqrt{\rho / \rho_{c}}$ given in (24),

$$
\frac{\Delta V}{V} \cdot \frac{\Delta(\sin \lambda \beta)}{\sin \lambda \beta} \geq 2 \pi G \hbar \gamma \lambda \frac{\sqrt{\rho_{c}} \cos \lambda \beta}{\sqrt{\rho_{o}} V^{(2-n) / 2}},
$$

where $\rho_{o}=V_{o}^{n} \tilde{\rho_{o}}$. It is easy to check that while the lefthand side of (35) is independent of $\mathcal{V}$, the right-hand side goes as $1 / V_{o}$.

Furthermore, bounding the $\cos \lambda \beta$ term by 1 , the strongest lower bound on the relative uncertainties occurs for the smallest value of $V$ that is reached, which is the volume of the fiducial cell at the bounce $V_{\text {bounce }}$. At the bounce point, the effective theory predicts that $\rho=\rho_{c}$, which implies that $\rho_{o}=\rho_{c} \cdot V_{\text {bounce. }}^{n}$ Therefore the strongest lower bound on the relative uncertainties is

$$
\frac{\Delta V}{V} \cdot \frac{\Delta(\sin \lambda \beta)}{\sin \lambda \beta} \geq \frac{2 \pi G \hbar \gamma \lambda}{V_{\text {bounce }}} .
$$

This shows that so long as the physical volume of the fiducial cell at the bounce point is significantly larger than the Planck volume, the relative uncertainties will never become large for an initially sharply-peaked state. Note however that if one chooses a fiducial cell so that $V_{\text {bounce }} \geq 2 \pi \gamma \ell_{\mathrm{Pl}}^{2} \lambda$ (recall $\lambda \sim \ell_{\mathrm{Pl}}$ ), then quantum fluctuations will become important when $V \sim 2 \pi \gamma \ell_{\mathrm{Pl}}^{2} \lambda$ at which point the effective equations will no longer be reliable.

Furthermore, it is also clear how this result generalizes to compact space-times: so long as the physical volume of the spatial slice remains much larger than the Planck volume, the relative uncertainties will not become important and the effective equations will be accurate.

\section{CONCLUSIONS}

We have concentrated on the flat FLRW model, but the results presented here can easily be generalized to other homogeneous cosmologies, whether the spatial manifold is compact or non-compact. This indicates that, for sharply peaked states, quantum back-reaction effects are negligible and the effective equations derived for the loop quantum cosmology of the Bianchi space-times and the Kantowski-Sachs space-times should be an excellent approximation to the quantum dynamics, so long as all length scales in the space-time remain much larger than the Planck length at all times.

These results can also be applied to cosmological perturbation theory. One way to study perturbations (up to some minimal wavelength) is by dividing the spatial manifold into a lattice of cells, where each cell is taken to be homogeneous. As the gravitational and matter fields vary from cell to cell, the fields are inhomogeneous at scales larger than the size of the cells. If the fields from one cell to another vary slightly around some mean value (which is taken to be the homogeneous background), then this setting can be used to study cosmological perturbations 13. Since each cell is assumed to be homogeneous, the arguments given here indicate that effective 
equations can be trusted (again assuming sharply peaked states) so long that the physical volume of each cell remains significantly larger than the Planck volume. As the minimal wavelength captured by this lattice is given by (twice) the cube root of the volume of each cell, this corresponds to modes whose wavelengths are much larger than the Planck length at all times. Therefore, the effective equations will be valid for such modes; however, they cannot be expected to be a good approximation for trans-Planckian modes where quantum fluctuations will necessarily be important.

This example further emphasizes that the choice of the fiducial cell must be made by taking into account the physics of interest. If one wishes to study the dynamics of the scale factor, the mean space-time curvature, the mean energy density and other large-scale observables that are typically of interest in minisuperspace models, it is appropriate to choose a very large fiducial cell and, for non-compact spaces, even take the limit of $V_{o} \rightarrow \infty$. On the other hand, this analysis has also shown that quantum fluctuations will necessarily be important for
trans-Planckian physics, and we do not expect effective equations to be reliable in that setting.

In short, local quantum fluctuations of the metric are not captured by minisuperspace models. The scale factor is a global quantity where the local quantum fluctuations are largely averaged out. This is why, for states that are initially sharply peaked, and so long as the volume of the region under consideration remains much larger than $\ell_{\mathrm{Pl}}^{3}$, relative quantum fluctuations can be arbitrarily small in minisuperspace models such as LQC.

\section{Acknowledgments}

We thank Parampreet Singh for helpful discussions. This work was supported in part by a grant from the John Templeton Foundation. The opinions expressed in this publication are those of the authors and do not necessarily reflect the views of the John Templeton Foundation.
[1] V. Taveras, "Corrections to the Friedmann Equations from LQG for a Universe with a Free Scalar Field," Phys. Rev. D78 (2008) 064072, arXiv:0807.3325

[2] P. Singh and V. Taveras, "A note on the effective equations in LQC," to be published.

[3] M. Bojowald, "Quantum Cosmology: Effective Theory," Class. Quant. Grav. 29 (2012) 213001, arXiv:1209.3403.

[4] A. Ashtekar, T. Pawłowski, and P. Singh, "Quantum Nature[10] of the Big Bang: Improved dynamics," Phys. Rev. D74 (2006) 084003, arXiv: gr-qc/0607039

[5] A. Corichi and E. Montoya, "Coherent semiclassical states for loop quantum cosmology," Phys. Rev. D84 (2011) 044021, arXiv: 1105.5081

[6] A. Ashtekar, M. Bojowald, and J. Lewandowski, "Mathematical structure of loop quantum cosmology," Adv. [1 Theor. Math. Phys. 7 (2003) 233-268, arXiv: gr-qc/0304074

[7] S. Holst, "Barbero's Hamiltonian derived from a generalized Hilbert-Palatini action," Phys. Rev. D53 (1996) 5966-5969,
arXiv: gr-qc/9511026

[8] A. Corichi and E. Montoya, "On the Semiclassical Limit of Loop Quantum Cosmology," Int. J. Mod. Phys. D21 (2012) 1250076, arXiv:1105.2804

[9] A. Ashtekar, A. Corichi, and P. Singh, "Robustness of key features of loop quantum cosmology," Phys. Rev. D77 (2008) 024046, arXiv:0710.3565

0] P. Singh and E. Wilson-Ewing, "Quantization ambiguities and bounds on geometric scalars in anisotropic loop quantum cosmology," Class. Quant. Grav. 31 (2014) 035010, arXiv: 1310.6728

[11] P. Diener, B. Gupt, and P. Singh, "Chimera: A hybrid approach to numerical loop quantum cosmology," Class. Quant. Grav. 31 (2014) 025013, arXiv:1310.4795

12] D. Poulin, "Macroscopic Observables," Phys. Rev. A71 (2005) 022102, arXiv:quant-ph/0403212

[13] E. Wilson-Ewing, "Lattice loop quantum cosmology: scalar perturbations," Class. Quant. Grav. 29 (2012) 215013, arXiv: 1205.3370. 